EPJ manuscript No.

(will be inserted by the editor)

\title{
Nuclear matter with off-shell propagation
}

\author{
P. Bożek \\ Institute of Nuclear physics, PL-31-342 Cracow, Poland
}

October 29, 2018

\begin{abstract}
Symmetric nuclear matter is studied within the conserving, self-consistent $T$-matrix approximation. This approach involves off-shell propagation of nucleons in the ladder diagrams. The binding energy receives contributions from the background part of the spectral function, away form the quasiparticle peak. The Fermi energy at the saturation point fulfills the Hugenholz-Van Hove relation. In comparison to the Brueckner-Hartree-Fock approach, the binding energy is reduced and the equation of state is harder.
\end{abstract}

Key words. nuclear matter - saturation point - thermodynamic properties - Hugenholz-Van Hove theorem

PACS. $21.65+\mathrm{f}$ Nuclear matter

The calculation of nuclear matter properties from the basic nucleon-nucleon interaction has been extensively studied using Brueckner type resummation of ladder diagrams. This resummation allows to rewrite the ground-state energy of nuclear matter using as an effective interaction, the $G$ matrix, which takes care of the short range re' pulsive core in the nucleon-nucleon interaction [1]. Calculations using realistic interactions lead to results, which lie along a line (the Coester line) shifted with respect to the phenomenological saturation point $\left(\rho_{0} \simeq .16 \mathrm{fm}^{-3}\right.$, $E / N \simeq-16 \mathrm{MeV}$ ). The remaining discrepancy can be attributed to relativistic effects and three-body forces contributions [2].

The results on the binding energy depend on the single particle energies used in the kernel of the Bethe-Goldstone equation [3]. The so called standard choice uses a selfconsistent auxiliary potential defined by the $G$-matrix below the Fermi energy and the free dispersion relation above $k_{F}$. Another choice is to use the self-consistent potential also above the Fermi momentum which gives the so called continuous choice for the single-particle energies in the Bethe-Goldstone equation. In Brueckner-HartreeFock (BHF) calculations the hole line expansion, irrespective of the choice of the auxiliary potential, is believed to converge to values close to the BHF with the continuous choice for single-particle energies [4].

Recently self-consistent approaches based on the in medium $T$-matrix approximation for nuclear matter have been studied [5, 6, 8, 8, 9, 10]. In this way a spectral function for nucleons in nuclear matter including two-particle correlations is obtained. The ladder diagrams involved in the calculation of the in medium $T$ matrix include also holehole propagation. The $T$-matrix approximation takes into account some of the higher order hole line contributions as compared to the $G$-matrix approach. It would be instructive to study the saturation properties of nuclear matter for the self-consistent $T$-matrix approximation with realistic interactions.

The $T$-matrix approach is a $\Phi$-derivable approximation 111. The self-energy is constructed as a functional derivative of a set of two-particle irreducible diagrams. This assures the fulfillment of thermodynamical relations for the quantities obtained [9]. The most famous such a relation is the equality of the Fermi energy and binding energy at the saturation point [12]

$$
E_{F}=E / N \text {. }
$$

The realization of the above relation is very important since it would give confidence to the single particle properties obtained in the calculations. In Ref. [9] we studied the self-consistent $T$-matrix approximation with a simple interaction confirming to a very good accuracy the fulfillment of thermodynamical relations by the numerical solutions. In BHF calculations the Hugenholz-Van Hove relation is badly violated. This discrepancy can be reduced by invoking rearrangement terms for the Fermi energy [13, 14, 15. By construction, the single-particle energies obtained in the $T$-matrix approximation come out consistently with thermodynamical observables. Thus we expect that singleparticle energies, scattering width or spectral functions directly obtained from the self-consistent $T$-matrix approximation are meaningful [10].

For attractive interactions cold nuclear matter forms a superfluid. Calculations using dressed propagators in the superfluid phase show a strong reduction of the gap [7, 16, 17. We expect that around the saturation point the superfluidity is very weak [16]. This means that the correction from the superfluid correlation energy to the binding en- 
ergy is small. We restrict ourselves to normal nuclear matter for all densities studied here. It allows us to compare with BHF calculations which are performed exclusively in the normal phase of nuclear matter.

The results here presented are obtained using a separable parameterization of the Paris potential [18 for $S$, $P, D$ and $F$ partial waves, for symmetric nuclear matter. We use rank 3 and rank 4 parameterization for the ${ }^{1} S_{0}$ and ${ }^{3} S_{1}-{ }^{3} D_{1}$ partial waves. In the ${ }^{3} P_{0}$ partial wave we use Mongan I interaction, in order to avoid unphysical resonances far off-shell. In the numerical iteration the full spectral function is discretized. For momenta close to the Fermi momentum the spectral function is separated into a background part and a quasiparticle peak approximated by a delta function. The numerical treatment of the energy integrations for the spectral functions is done using convolution algorithms [10].

The $T$-matrix approximation resumes ladder diagrams with dressed particle-particle and hole-hole propagators

$$
\begin{aligned}
& <\mathbf{p}|T(\mathbf{P}, \Omega)| \mathbf{p}^{\prime}>=V\left(\mathbf{p}, \mathbf{p}^{\prime}\right) \\
& +\int \frac{d \omega_{1}}{2 \pi} \int \frac{d \omega_{2}}{2 \pi} \int \frac{d^{3} q}{(2 \pi)^{3}} V(\mathbf{p}, \mathbf{q}) \frac{\left(1-f\left(\omega_{1}\right)-f\left(\omega_{2}\right)\right)}{\Omega-\omega_{1}-\omega_{2}+i \epsilon} \\
& A\left(p_{1}, \omega_{1}\right) A\left(p_{2}, \omega_{2}\right)<\mathbf{q}|T(\mathbf{P}, \Omega)| \mathbf{p}^{\prime}>
\end{aligned}
$$

where $\mathbf{p}_{\mathbf{1}, \mathbf{2}}=\mathbf{P} / 2 \pm \mathbf{q}$ and $f(\omega)$ is the Fermi distribution. The imaginary part of the corresponding retarded selfenergy can be obtained closing a pair of external vertices in the $T$-matrix with a fermion propagator

$$
\begin{aligned}
& \operatorname{Im} \Sigma(p, \omega)=\int \frac{d \omega_{1}}{2 \pi} \int \frac{d^{3} k}{(2 \pi)^{3}} A\left(k, \omega_{1}\right) \\
& <(\mathbf{p}-\mathbf{k}) / 2\left|\operatorname{Im} T\left(\mathbf{p}+\mathbf{k}, \omega+\omega_{1}\right)\right|(\mathbf{p}-\mathbf{k}) / 2>_{A} \\
& \left(f\left(\omega_{1}\right)+b\left(\omega+\omega_{1}\right)\right)
\end{aligned}
$$

where

$$
A(p, \omega)=\frac{-2 \operatorname{Im} \Sigma(p, \omega)}{\left(\omega-p^{2} / 2 m-\operatorname{Re} \Sigma(p, \omega)\right)^{2}+\operatorname{Im} \Sigma(p, \omega)^{2}}
$$

is the self-consistent spectral function of the nucleon and $b(\omega)$ is the Bose distribution. The real part of the selfenergy is related to $\operatorname{Im} \Sigma$ by a dispersion relation

$$
\operatorname{Re} \Sigma(p, \omega)=\Sigma_{H F}(p)+\mathcal{P} \int \frac{d \omega^{\prime}}{\pi} \frac{\operatorname{Im} \Sigma\left(p, \omega^{\prime}\right)}{\omega^{\prime}-\omega}
$$

with $\Sigma_{H F}(p)$ the Hartree-Fock self-energy. Eqs. 2, 3, 5 and 4 are to be solved iteratively and at each iteration the chemical potential $\mu$ is adjusted to fulfill the condition on the density $\rho$

$$
\int \frac{d \omega}{2 \pi} \int \frac{d^{3} p}{(2 \pi)^{3}} A(p, \omega) f(\omega)=\rho .
$$

The spectral functions obtained in the self-consistent solution consist of a quasiparticle peak and a broad background (Fig. 1). As function of momentum the position

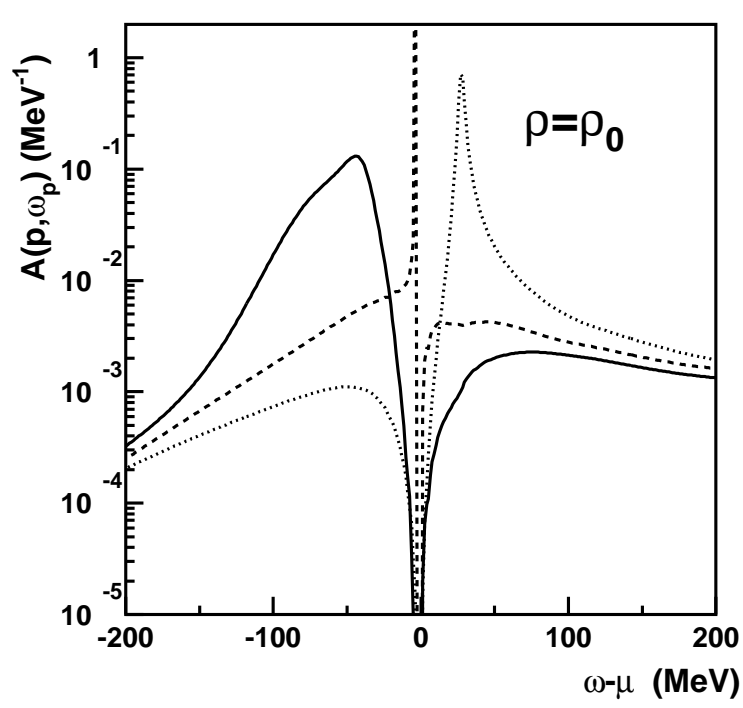

Fig. 1. The spectral function $A(p, \omega)$ as function of energy for $p=0,255$ and $340 \mathrm{MeV}$ (solid, dashed, and dotted lines respectively).

of the peak in the spectral function follows approximately the quasiparticle dispersion relation

$$
\omega_{p}=\frac{p^{2}}{m}+\operatorname{Re} \Sigma\left(p, \omega_{p}\right)
$$

The quasiparticle peak is very sharp for momenta close to the Fermi momentum. It is a manifestation of the quasiparticle nature of excitations close to the Fermi surface. Indeed we find that the single-particle width $-2 \operatorname{Im} \Sigma\left(p, \omega_{p}\right)$ is proportional to $\left(p-p_{F}\right)^{2}$ close to the Fermi momentum. The background of the spectral functions extend far from the quasiparticle peak. The part of the spectral function below the Fermi energy leads to nonzero occupancy for momenta above $p_{F}$ and gives a large, negative contribution to the binding energy for all momenta.

The nucleon momentum distribution

$$
n(p)=\int \frac{d \omega}{2 \pi} A(p, \omega) f(\omega)
$$

is very different different from the Fermi-Dirac distribution. Momentum states below the Fermi momentum are depleted and a tail in the distribution $n(p)$ for large momenta appears. The $T$-matrix approximation leads to a Fermi liquid behavior in the normal phase, with a jump in the fermion density of $Z_{p_{F}}=\left(1-\left.\frac{\partial \operatorname{Re} \Sigma\left(p_{F}, \omega\right)}{\partial \omega}\right|_{\omega=E_{F}}\right)^{-1} \simeq$ .74 at the Fermi momentum. In the calculation the Fermi momentum is fixed by the constraint (6) on the total density. For a conserving approximation the Fermi momentum should be the same as the Fermi momentum of a free 


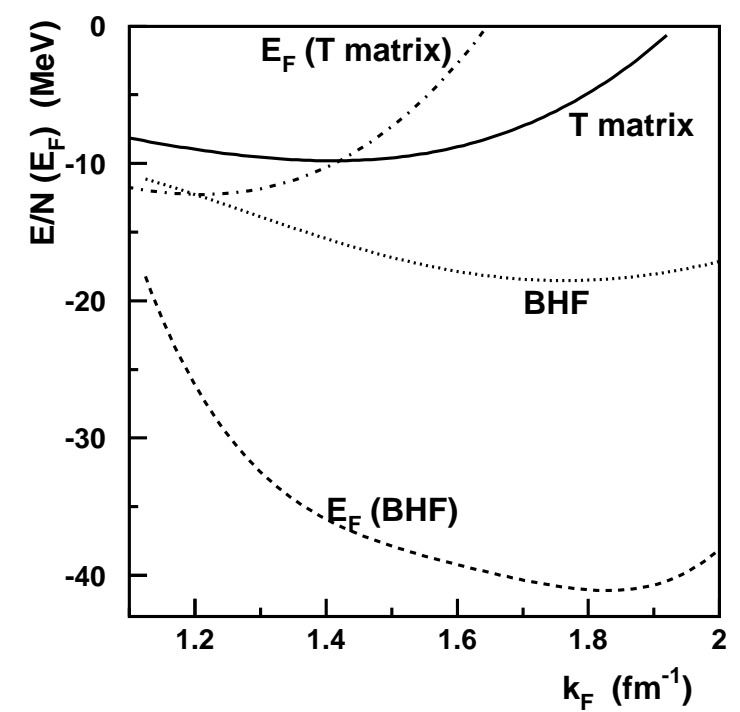

Fig. 2. The binding energy for the $T$-matrix (solid line) and for the BHF (dotted line) calculations, and the Fermi energy for the $T$-matrix (dashed-dotted line) and for the BHF (dashed line) calculations as function of the Fermi momentum.

fermion gas 19, 11]. This thermodynamical consistency relation is verified to a good accuracy by our calculations for the range of densities studied.

The energy per particle, in the case of only two-body interactions, can be obtained from the single-particle spectral function

$$
E / N=\frac{1}{\rho} \int \frac{d^{3} p}{(2 \pi)^{3}} \frac{d \omega}{2 \pi} \frac{1}{2}\left(\frac{p^{2}}{2 m}+\omega\right) A(p, \omega) f(\omega) .
$$

The binding energy per nucleon as function of the Fermi momentum is presented in Fig. 2 for the self-consistent $T$-matrix approximation and compared to results from $G$ matrix calculations using the continuous choice of the auxiliary potential. The results of the $T$-matrix approach lie above to the BHF binding energy for densities close to the phenomenological saturation point. Since we know that further hole line corrections do not modify the continuous BHF results drastically, we get an assessment of the accuracy of the $T$-matrix approach. The higher the density the larger the discrepancy becomes. Correspondingly the saturation point in the $T$-matrix approach is shifted to lower densities $\left(\rho=1.4 \rho_{0}\right.$ instead of 2.4 $\left.\rho_{0}\right)$ and lower binding energies (the binding energy is reduced by $4 \mathrm{MeV}$ at $\rho_{0}$ ). Very similar results are found for the equation of state of pure neutron matter [17]. We note the the Hugenholz-Van Hove condition (1i) is very well satisfied.

The origin of the of the binding energy in the $T$-matrix approximation can be understood writing Eq. (9) as

$$
E / N=\frac{1}{\rho} \int \frac{d^{3} p}{(2 \pi)^{3}} n(p) \frac{1}{2}\left(\frac{p^{2}}{2 m}+\overline{\omega_{p}}\right)
$$

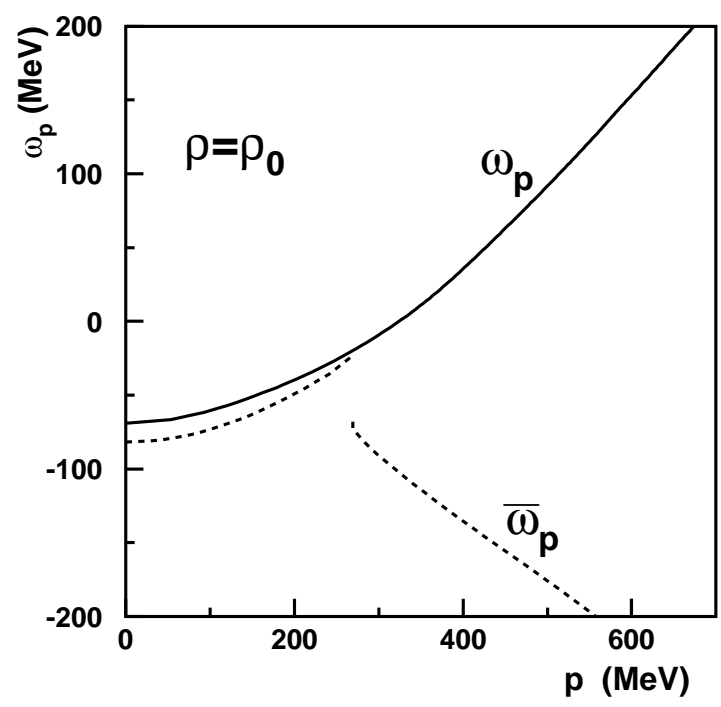

Fig. 3. The quasiparticle energy $\omega_{p}$ and the average energy $\bar{\omega}_{p}$ (11) as function of momentum.

with

$$
\overline{\omega_{p}}=\int \frac{d \omega}{2 \pi} \omega A(p, \omega) f(\omega) / n(p),
$$

whereas in quasiparticle approaches it is

$$
E / N=\frac{1}{\rho} \int \frac{d^{3} p}{(2 \pi)^{3}} n(p) \frac{1}{2}\left(\frac{p^{2}}{2 m}+\omega_{p}\right) .
$$

In Fig. 3 we compare the removal energy $\overline{\omega_{p}}$ to the quasiparticle energy $\omega_{p}$. Due to a large contribution of the background strength of the spectral function lying below the quasiparticle peak the removal energy is much below the quasiparticle energy. On the other hand, the singleparticle energy $\omega_{p}$ in the $T$-matrix approximation is generally above the one obtained from $G$-matrix calculations. It can be seen by comparing the Fermi energies in the two approximations in Fig. 2. These differences explain why the Fermi energy in the conserving $T$-matrix approximation is equal to the binding energy at the local saturation point, following the Hugenholz-Van Hove relation (11). The binding energy is determined by $\overline{\omega_{p}}$ and $E_{F}=\omega_{p_{F}}$ for the $T$-matrix calculation, whereas both quantities are determined by $\omega_{p}$ in the $G$-matrix scheme.

This paper presents the first results self-consistent $T$ matrix calculation of saturation properties of nuclear matter with a realistic potential. The binding energy obtained is smaller than the BHF result with the continuous auxiliary potential. We note that a very similar shift in binding energy is observed in a BHF calculation, when including the rearrangement terms contribution to the binding energy [15]. In Ref. [15] by considering rearrangement term corrections to the single particle energies and to the binding energy an improvement of the fulfillment Hugenholz- 
P. Bożek: Nuclear matter with off-shell propagation

Van Hove relation is found. The same can be observed in the $T$-matrix approach, we destroy a bit the binding energies and improve considerably the single particle energies from the BHF approach to get the relation (1i) right. We expect that after inclusion of ring diagrams contributions, as well as higher partial waves and three body forces corrections, the results on the saturation properties of nuclear matter of modern BHF approaches will be recovered. The calculation of these corrections is standard and not related to the $T$-matrix approach. On the other hand, the real advantage of the self-consistent $T$-matrix approximation shows itself in the single-particle properties. The quasiparticle energies lead to a Fermi energy consistent with the Hugenholz-Van Hove relation. The spectral function for large negative and positive energies can be calculated, with implications for the binding energy and applications for electron scattering. We find at normal nuclear density an effective mass $m^{\star}=.9 \mathrm{~m}$ and $Z_{p_{F}}=.74$. These numbers can be used in the estimation of reduced in medium cross sections and density of states at the Fermi energy. Generally, $Z_{p}$ does not change much with density or with the details of the potential used, provided the lowest partial waves are described reasonably. We confirm the thermodynamical consistency of the numerical solution of the $T$-matrix scheme 90 for realistic interaction with several partial waves also. Finally let us note that the self-consistent $T$-matrix calculation can be straightforwardly extended to finite temperatures.

This work was partly supported by the KBN under Grant No. 2P03B02019.

\section{References}

1. K.A. Brueckner and J.L. Gammel, Phys. Rev. 109, 1023 (1958); B.D. Day, Rev. Mod. Phys. 39719 (1967) J.P. Jeukenne, A. Leugeunne and C. Mahaux, Phys. Rep. 25, 83 (1976).

2. R. Brockmann and R. Machleit, Rhys. Rev. C42, 1965 (1990).

3. R.B. Wiringa, R.A. Smith and T.L. Ainsworth, Phys. Rev. C29, 1207 (1984).

4. H.Q. Song, M. Baldo, G. Giansiracusa and U. Lombardo, Phys. Rev. Lett. 811584 (1998).

5. W.H. Dickhoff, Phys. Rev. C58 2807 (1998); W.H. Dickhoff, C.C. Gearhart, E.P. Roth, A. Polls and A. Ramos, Phys. Rev. C60 4319 (1999).

6. P. Bożek, Phys. Rev. C59 2619 (1999).

7. P. Bożek, Nucl. Phys. A657 187 (1999); P. Bożek, Phys. Rev. C62, 054316 (2000).

8. Y. Dewulf, D. Van Neck and M. Waroquier, Phys. Lett. B510, 89 (2001).

9. P. Bożek and P. Czerski, Eur. Phys. J. A11, 271 (2001).

10. P. Bożek, nucl-th/0201086.

11. G. Baym, Phys. Rev. 1271392 (1962).

12. N.M. Hugenholz and L. Van Hove, Physica 24363 (1958).

13. M. Baldo, F. Bombaci, G. Giansiracusa, U. Lombardo, C. Mahaux and R. Sartor,, Phys. Rev. C41 1748 (1990).

14. F. de Jong and R. Malfliet, Phys. Rev. C44 998 (1991).

15. P. Czerski, A. De Pace and A. Molinari, nucl-th/0204008.
16. P. Bożek, nucl-th/0202045.

17. P. Bożek and P. Czerski, nucl-th/0204012.

18. J. Haidenbauer and W. Plessas, Phys. Rev C30, 1822 (1984); ibid, C32, 1424 (1985).

19. J.M. Luttinger and J.C. Ward, Phys. Rev. 1181417 (1960); J.M. Luttinger, Phys. Rev. 1191153 (1960). 